\title{
HCV cure without interferon
}

\author{
Marc Bourlière \\ From International Symposium HIV and Emerging Infectious Diseases 2014 \\ Marseille, France. 21-23 May 2014
}

The launch of first-generation protease inhibitors (PIs) was a major step forward in HCV treatment. However, this major advance was restricted to genotype 1 (GT-1) patients. This year the launch of Sofosbuvir a NS5B nucleotide inhibitor (Nis), Daclatasvir a NS5A inhibitor (NS5A.I) and Simeprevir a second wave PIs open new perspective for IFN-free regimen. Both Sofosbuvir and Daclatasvir have a pan-genotypic activity. Sofosbuvir has highly potent antiviral activity across all genotypes in association with pegylated interferon and ribavirin (PR), thus allowing shortened treatment duration. Moreover, Sofosbuvir in combination with Daclatasvir without ribavirin for 12 weeks was able to cure $>95 \%$ of naïve GT-1 and for 24 weeks was able to cure 40 patients with treatment experienced, failure to triple therapy with first generation PIs, GT-1 patients. Sofosbuvir in combination with Simeprevir for 12 weeks without ribavirin was able to cure all GT-1 naïve patients and over $90 \%$ of treatment experienced GT-1 patients. As the three drugs have potent antiviral activities against GT-4, such association must be potent in such patient but data are pending.

For GT-2, Sofosbuvir and ribavirin for 12 weeks are able to cure $>90 \%$ of naïve and treatment-experienced patients. For GT-3 patients, Sofosbuvir and ribavirin for 24 weeks are able to cure around $90 \%$ of naïve and treatment experienced patients with the exception of treatment-experienced cirrhotic patients in whom SVR is around $60 \%$. However the combination of Sofosbuvir and Daclatasvir for 24 week leads to more than $90 \%$ SVR in the naïve GT-3 population and trials are on-going for treatment-experienced patients. All this data confirmed that $\mathrm{HCV}$ can be cure with interferon-free regimen and short duration of treatment. Other combination with higher efficacy and very good safety profile are under development and should be available within two years.

Hepato-Gastroenterology Department, Saint Joseph Hospital, Marseille, 13285, France

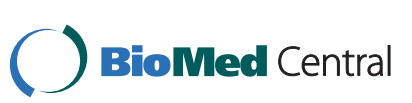

(c) 2014 Bourlière; licensee BioMed Central Ltd. This is an Open Access article distributed under the terms of the Creative Commons Attribution License (http://creativecommons.org/licenses/by/4.0), which permits unrestricted use, distribution, and reproduction in any medium, provided the original work is properly cited. The Creative Commons Public Domain Dedication waiver (http:// creativecommons.org/publicdomain/zero/1.0/) applies to the data made available in this article, unless otherwise stated. 